\title{
Myocardial abscess with complete heart block complicating anaerobic infective endocarditis
}

\author{
HARRY A KOPELMAN, BARNEY S GRAHAM, MERVYN B FORMAN \\ From the Departments of Medicine and Pathology, Division of Cardiology and Infectious Disease, Vanderbilt \\ University Medical Center, Nashville, Tennessee, USA
}

SUMMARY Myocardial abscess caused by anaerobic infection is rare and usually occurs in cases of myocardial infarction, in which it may be related to areas of low oxygen tension. Bacteroides CDC group F-1 infective endocarditis complicated by an aortic valve ring abscess with resultant complete heart block developed in a patient with steroid dependent systemic lupus erythematosus. The genitourinary system was the presumed source of the infection. Endocarditis developed after an elective abortion, despite antibiotic prophylaxis according to American Heart Association recommendations. This case shows that an anaerobic abscess of the aortic valve ring can affect contiguous vital structures of the conducting system. Immunosuppression may increase the risk of anaerobic infection after genitourinary procedures, and in this situation the recommended antibiotic prophylaxis may be inadequate.

Myocardial abscess caused by anaerobic infection is rare. ${ }^{1-8}$ Most cases have occurred after myocardial infarction, and none was associated with conduction system disturbances. We report a case in which a Bacteroides CDC group F-1 organism caused infective aortic valve endocarditis, myocardial abscess, and complete heart block. This organism has not been previously reported as a cause of infective endocarditis. The case demonstrates the difficulty and importance of recognising anaerobic infections of the heart.

\section{Case report}

A 23 year old black woman with systemic lupus erythematosus presented with a two week history of fever, chills, chest pain, myalgia, and gradually worsening dyspnoea. Systemic lupus erythematosus had been diagnosed eight years earlier on the basis of American Rheumatology Association criteria, which included polyserositis, non-deforming polyarthritis, biopsy proven membranous glomerulonephritis, positive lupus erythematosus cells, $4+$ antinuclear antibodies with rim pattern, and high titres of

Requests for reprints to Dr Mervyn B Forman, Division of Cardiology, Vanderbilt University School of Medicine, Nashville, Tennessee 37232, USA. double stranded DNA. She had been steroid dependent for five years before presentation.

The patient conceived 10 months before presentation but fetal death in utero occurred at approximately 36 weeks' gestation. Labour was induced by ultrasound guided transabdominal intraamniotic instillation of prostaglandin $F_{2}$. Antibiotic prophylaxis (including intravenous ampicillin and gentamicin) was given according to American Heart Association guidelines. ${ }^{9}$ She was discharged feeling well on maintenance prednisone of $10 \mathrm{mg} /$ day.

Two weeks before presentation she was readmitted with a two day history of fever, chills, chest pain, and myalgia. A new diastolic murmur of aortic regurgitation was noted and an electrocardiogram showed non-specific ST-T wave changes (Fig. 1a). She improved after six days of intravenous cefazolin sodium, tobramycin, and methylprednisolone. Six sets of blood cultures yielded no growth and she was discharged on prednisone $40 \mathrm{mg} /$ day.

On admission to Vanderbilt University Medical Center, she was sweating and tachypnoeic, with a pulse rate of 120 beats/min and blood pressure of $140 / 70 \mathrm{~mm} \mathrm{Hg}$. The apical impulse was hyperdynamic and the intensity of the first heart sound was reduced. A grade 3/6 early diastolic murmur was present at the left sternal edge, and a grade $2 / 6$ (ejection) systolic murmur conducted into the carotids. Chest $x$ ray showed cardiomegaly with left 


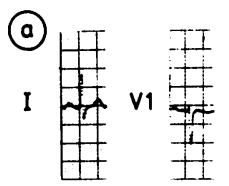

(b)

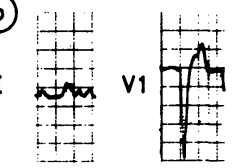

II

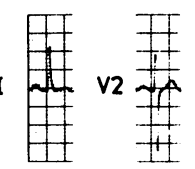

III

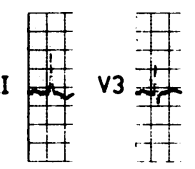

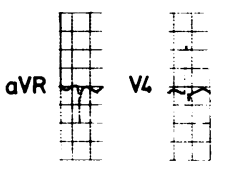
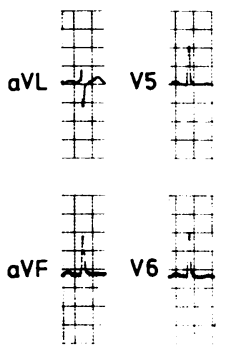

II

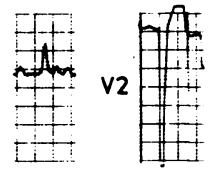

III

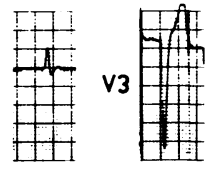

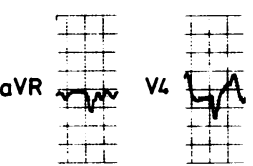
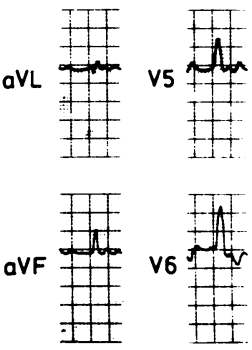

Fig. 1 (a) Baseline electrocardiogram shows non-specific $S T-T$ wave changes. (b) On presentation prolongation of $P R$ interval was associated with complete left bundle branch block.

ventricular prominence and upper lobe blood diversion.

The electrocardiogram (Fig. 1b) showed sinus tachycardia with new $P-R$ interval prolongation and left bundle branch block. A cross sectional echocardiogram demonstrated a bulky aortic valve vegetation which prolapsed into the left ventricular outflow tract during diastole. The mitral valve leaflets were diffusely thickened with no discrete vegetations. Blood cultures were obtained, and she was started on intravenous ampicillin, oxacillin, and gentamicin. Complete heart block developed within six hours and her condition worsened. During preparation for emergency cardiac operation she suffered a cardiopulmonary arrest. At operation dehiscence of the left and non-coronary aortic valve cusps with an extensive aortic perivalvar abscess infiltrating the

interventricular septum was found. A Björk-Shiley prosthetic valve was inserted, but the patient had $c$ suffered anoxic brain damage and she died on the $\overrightarrow{\vec{F}}$ sixth hospital day.

Bacteroides CDC group F-1 was grown from the intraoperative culture of the myocardial abscess and $\overline{\bar{c}}$ identified by the Tennessee Department of Health $\vec{\nabla}$ and Environment, Laboratory Services. Twelve sets of blood cultures, including anaerobic cultures, yielded no growth.

\section{POSTMORTEM FINDINGS}

The heart weighed $550 \mathrm{~g}$ and there was concentric left ventricular hypertrophy. Postmortem injection of of barium-gelatin dye demonstrated normal epicardial coronary arteries and no evidence of athero- $\overrightarrow{.}$ sclerosis. At necropsy, however, there was sub- $\vec{\circ}$ endocardial infarction of the lateral wall of the left 0

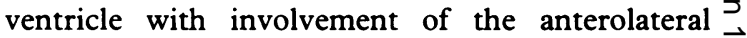
papillary muscle. There was no histological evidence $c$ of vasculitis or myocarditis. A Björk-Shiley valve $\stackrel{\sum}{\sum}$

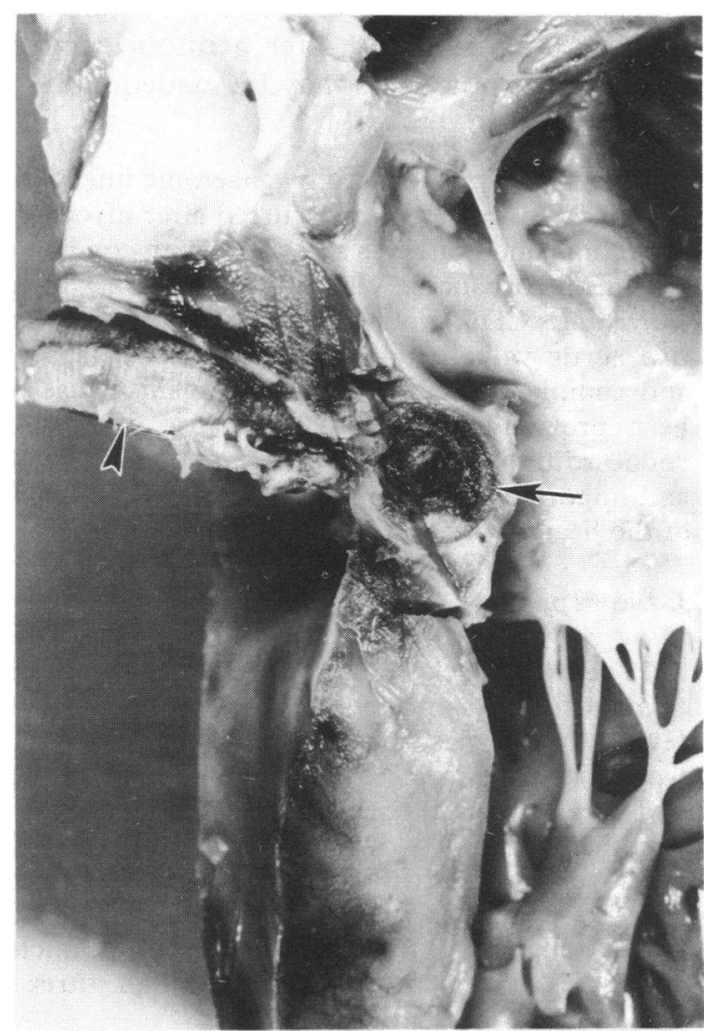

Fig. 2 Transverse section of the heart showing perivalvar abscess in the membranous septum (arrow). The stent of the Björk-Shiley valve is present in the aortic position (arrowhead). 
was in place in the aortic position and haemorrhage was noted around the annulus. A perivalvar abscess was present at the base of the aorta and extended through the interventricular septum to involve the superior border of the septal leaflet of the tricuspid valve (Fig. 2). The posterior mitral valve leaflet was shortened, thickened, and adhered to the left ventricular posterior wall. The surgically excised aortic valve showed a large friable vegetation with perforation of the anterior leaflet.

\section{Discussion}

\section{MYOCARDIAL ABSCESS WITH COMPLETE}

HEART BLOCK

The non-coronary sinus of Valsalva lies close to the superior interventricular septum and His bundle. ${ }^{1011}$ Usually the left bundle branch runs in the base of the membranous septum or along the left side of the crest of the interventricular septum; however, Massing and James noted that in five of 32 necropsy specimens it traversed the right side of the interventricular septum. ${ }^{10}$ In those cases the left bundle branch calibre was unusually small (mean cross sectional area $1.5 \mathrm{~mm}^{2}$ ), and because of their increased length these branches were particularly susceptible to injury.

These anatomical relations explain why aortic perivalvar abscesses that affect the posterior aortic sinus, causing mycotic aneurysm and cardioaortic fistulas, account for most cases of complete heart block in acute infective endocarditis. ${ }^{1213}$ Complete heart block occurs in 0-14\% cases of infective endocarditis. ${ }^{14-16}$ In 18 of the 20 cases of atrioventricular conduction disturbances reported by Wang et al the aortic valve was affected; these included all six cases of complete heart block. ${ }^{12}$ Extension of infection into the conduction system accounted for complete heart block in all of them. When the aortic valve is infected, myocardial abscess is suggested by high grade atrioventricular block, aortic regurgitation, pericarditis, and rapid clinical deterioration. ${ }^{13}$ The presence of several of these features in our case led to the diagnosis of myocardial abscess. The development of complete heart block signalled a high risk infection that required immediate operation. ${ }^{17}$

\section{ANAEROBIC ENDOCARDITIS AND MYOCARDIAL ABSCESS}

Anaerobic infections are responsible for only $2-16 \%$ of all reported cases of endocarditis. ${ }^{18-20}$ With three exceptions the presenting symptoms and signs are the same as those of infective endocarditis caused by facultative aerobic organisms. Patients with anaerobic endocarditis have a lower frequency of pre-existing valvar heart disease $\left(48-64 \%{ }^{18} 19\right.$ vs $\left.75-100 \%{ }^{14}{ }^{15}\right)$. Major thromboembolic events are more common and the mortality rate is higher because of more frequent complications and the difficulty of diagnosis and treatment. ${ }^{18-22}$ The twelve negative blood cultures obtained during our patient's three week illness confirm that anaerobes such as Bacteroides can be a cause of blood culture negative endocarditis. ${ }^{18}$

Although deep tissue abscesses are common in anaerobic bacteraemias or as an embolic complication of endocarditis, frank myocardial abscess is rare. ${ }^{1}$ Few documented cases have been reported, and these have usually been associated with myocardial infarction. ${ }^{1-8}$ It is likely that the locally reduced oxidation-reduction potential resulting from ischaemic necrosis and its byproducts promotes anaerobic infection and abscess formation.

Anaerobic aortic perivalvar abscess with septal extension causing complete heart block has not been reported before. This complication may have been promoted by abnormal host defences (long term steroid treatment and an autoimmune disorder). At necropsy a subendocardial myocardial infarction was noted at a site distant from the abscess, although the epicardial coronary arteries were normal. It is possible that in the setting of acute aortic insufficiency, myocardial ischaemia resulting from increased left ventricular end diastolic pressure, increased wall stress, and decreased coronary reserve provided the environment for anaerobic growth in the hypertrophied interventricular septum. There was no evidence of vasculitis, myocarditis, or coronary embolus.

\section{SOURCE OF BACTEROIDES ENDOCARDITIS}

Anaerobic gram negative non-spore forming bacilli are normally present in the human respiratory, gastrointestinal, and genitourinary tracts. Although the gastrointestinal tract is thought to be the most common portal of entry for Bacteroides, the genitourinary tract is another source. ${ }^{2122}$ Anaerobic bacteria predominate in the female genital tract, and transient bacteraemia, predominantly anaerobic, occurs in $85 \%$ of women undergoing suction abortion. ${ }^{23} 24$

Although Bacteroides CDC group F-1 is known to cause bacteraemia ${ }^{25}$ it has never been reported as a cause of infective endocarditis. This organism was grown from tissue obtained at cardiac operation in our patient.

IMPLICATIONS FOR ANTIBIOTIC PROPHYLAXIS AND TREATMENT

Our patient was given the antibiotic prophylaxis recommended for genitourinary tract operation or 
manipulation, ${ }^{9}$ but ampicillin and gentamicin prophylaxis did not prevent anaerobic endocarditis in this case. Animal studies have demonstrated that high dose clindamycin is more effective than other antibiotics against Bacteroides fragilis endocarditis. ${ }^{25}$ Metronidazole, which has not been tested as an agent for endocarditis prophylaxis, is perhaps the most powerful anti-anaerobic antimicrobial drug. It is safe; and high serum and tissue concentrations may be achieved with oral doses. ${ }^{26}$

Most failures of endocarditis prophylaxis are the result of not conforming to current American Heart Association recommendations ${ }^{27}$; this case is an exception and on the basis of just one case we cannot recommend that the standard antimicrobial prophylaxis for genitourinary tract manipulation should be expanded to include improved anaerobic cover. Nevertheless, in high risk patients with suspected endocarditis who have undergone such procedures, anaerobes should be considered as potential pathogens.

\section{References}

1 Finegold SM. Anaerobic bacteria in human disease. New York: Academic Press, 1977: 201-22.

2 Tennant R, Parks HW. Myocardial abscess. A study of pathogenesis with report of a case. Arch Pathol 1959; 68: 456-60.

3 Pittman DE, Merkow LP, Brent LB. Myocardial abscesses causing occlusion of the coronary ostium. Arch Intern Med 1970; 126: 294-7.

4 Lewis JF. Myocardial infarction during pregnancy with associated myocardial Bacteroides abscess. South Med f 1973; 66: 379-81.

5 Guneratne F. Gas gangrene (abscess) of heart. NY State f Med 1975; 75: 1766-9.

6 Esteban A, Wilson WR, Ruiz-Santanas S, FernandezSergovino P, Martinez Cabruja R. Endocarditis caused by $B$ fragilis. Chest $1983 ; 84: 104-7$.

7 Case Records of the Massachusetts General Hospital. Case 27-1970. N Engl f Med 1970; 282: 1477-85.

8 Finley RW, Marr JJ. Anaerobic myocardial abscess following myocardial infarction. Am $\mathcal{F}$ Med 1985; 78: 513-4.

9 Kaplan EL, Anthony BF, Bisno A, et al. AHA Committee Report: prevention of bacterial endocarditis. Circulation 1977; 56: 139A-43A.

10 Massing GK, James TN. Anatomical configuration of the His bundle and bundle branches in the human heart. Circulation 1976; 53: 609-21.
11 Kistin AD. Observations on the anatomy of the atrioventricular bundle (bundle of His) and the question of other muscular atrioventricular connections in normal human hearts. Am Heart $\mathcal{F}$ 1949; 37: 849-67.

12 Wang K, Gobel F, Gleason DF, Edwards JE. Complete heart block complicating bacterial endocarditis. Circulation 1972; 46: 939-47.

13 Arnett EN, Roberts WC. Valve ring abscess in active infective endocarditis: frequency, location and clues to clinical diagnosis from the study of 95 necropsy patients. Circulation 1976; 54: 140-5.

14 Pelletier LL, Petersdorf RG. Infective endocarditis: a review of 125 cases from the University of Washington Hospitals, 1963-1972. Medicine (Baltimore) 1977; 56: 287-313.

15 von Reyn CF, Levy BS, Arbeit RD, Friedland GF, Crumpacker CS. Infective endocarditis: an analysis based on strict case definitions. Ann Intern Med 1981; 94: 505-18.

16 Dinubile MJ. Heart block during bacterial endocarditis: a review of the literature and guidelines for surgical intervention. Am $\mathcal{F}$ Med Sci 1984; 287: 30-2.

17 Stinson EB. Surgical treatment of infective endocarditis. Prog Cardiovasc Dis 1979; 22: 145-69.

18 Felner JM, Dowell VR. Anaerobic bacterial endocarditis. $N$ Engl f Med 1970; 283: 1888-92.

19 Nastro LJ, Finegold SM. Endocarditis due to anaerobic gram-negative bacilli. Am F Med 1973; 54: 482-96.

20 Nord CE. Anaerobic bacteria in septicemia and endocarditis. Scand $\mathcal{F}$ Infect Dis 1982; 31 (suppl): 95-104.

21 Bodner SJ, Koenig MG, Goodman JS. Bacteremic bacteroides infections. Ann Intern Med 1970; 73: 537-44.

22 Felner JM, Dowell VR. "Bacteroides" bacteremia. Am f Med 1971; 50: 787-96.

23 Bartlett JG, Moon NE, Goldstein PR, Onderdonk AB, Polk BF. Cervical and vaginal bacterial flora: ecological niches in the female lower genital tract. Am $\mathcal{F}$ Obstet Gynecol 1978; 130: 658-61.

24 Ritvo R, Monroe P, Andrioble VT. Transient bacteremia due to suction abortion: implications for SBE antibiotic prophylaxis. Yale $\mathcal{F}$ Biol Med 1977; 50: 471-9.

25 Goldman P, Durack DT, Petersdorf RG. Effect of antibiotics on the prevention of experimental Bacteriodes fragilis endocarditis. Antimicrob Agents Chemother 1978; 14: 755-60.

26 Ralph ED, Kirby WM. Unique bactericidal action of metronidazole against Bacteroides fragilis and Clostridium perfringens. Antimicrob Agents Chemother 1975; 8: 409-14.

27 Durack DT, Kaplan EL, Bisno AL. Apparent failures of endocarditis prophylaxis. $\mathcal{J} A M A$ 1983; 250: 2318-22. 